\title{
Use of in-class physics demonstrations in highly interactive format
}

\author{
Kandiah Manivannan, Department of Physics, Astronomy, and Materials Science
}

Southwest Missouri State University, Springfield, MO 65804

David E. Meltzer, Department of Physics \& Astronomy, Iowa State University, Ames, IA 50011

We show how traditional classroom demonstrations may be converted into active-learning experiences through linked multiple-choice question-and-answer sequences. Sample question sequences and worksheet materials are presented, as well as preliminary assessment data.

\section{INTRODUCTION}

In-class demonstrations have been considered by physics instructors to be a very important part of teaching physics. Physics demonstrations have been around for many years, and physics teachers and researchers have written numerous articles and books on classroom demonstrations. Demonstrations can certainly make physics classes fun and entertaining, and they may also stimulate students' interest and curiosity. Surveys conducted for the Introductory University Physics Project indicate that students believe demonstrations help them to better visualize and think about physics.

However, despite these positive aspects of physics demonstrations, there is a growing body of evidence suggesting that traditional in-class demonstrations are not very effective in promoting conceptual understanding of physics. One important factor is the lack of active participation and interaction of students during physics demonstrations. Recent research studies indicate that students who saw traditional physics demonstrations in a course fared no better than students who did not see the demonstrations. $^{1,2}$ The data do suggest, however, that there is at least a small improvement in performance when students have to predict the outcome of a demonstration before seeing it. Based on these and other studies, it has become increasingly clear that some form of interactive engagement is essential to maximize the effectiveness of classroom demonstrations.

At this time there are relatively few research-based curricular materials available for physics demonstrations. The pioneering work of Sokoloff and Thornton ${ }^{3}$ on interactive lecture demonstrations (ILD) is probably the most comprehensive curricular material of that type available today. Their published results on ILDs indicate dramatic learning gains for students who were taught using ILDs compared to students who took a traditional course. ILDs require the use of Microcomputer-Based Laboratory equipment.

We have been developing new curricular materials on interactive physics demonstrations that would promote active learning in physics classes. Our goal is to produce activities that are suitable for any classroom setting and can easily be implemented without any additional resources or logistical support (such as computer hardware or teaching assistants). Our teaching strategy can be used with "hightech" demonstrations as well as with those that are low-tech. The central feature is the use of the problem-dissection technique ${ }^{4}$ to break a given physics demonstration into several conceptually linked minidemonstrations. The demonstrations are presented to the class in a sequence while utilizing techniques (such as "flash cards") for acquiring immediate feedback from all the students in the class simultaneously. We find this approach very effective in helping students construct a deeper understanding of physical concepts through step-by-step confrontation with their alternate conceptions. Since these innovative elements are based on findings of physics education research, one may hope that student learning might be significantly more effective than in a course taught using 
traditional methods. In this paper we report our preliminary findings on a specific interactive demonstration activity involving free-falling objects.

\section{METHOD}

The most important features of the interactive demonstration curricular materials are as follows:

1. The curricular materials or worksheets are designed to strongly promote studentstudent as well as student-faculty interaction in the classroom.

2. The initial prediction of the outcome of the demonstration and the subsequent discussion among neighboring students - as well as the following class-wide discussion - are very important parts of the demonstration activity.

3. Activities are based on the premise that the explanation of even a very simple physics demonstration invariably hinges on a lengthy chain of concepts and reasoning.

4. The problem-dissection technique ${ }^{4}$ is used to break a given physics demonstration into several conceptually linked minidemonstrations.

5. The mini-demonstrations are presented as a sequence in a pre-determined order. Breaking down the main demonstration into smaller component demonstrations is very effective in helping students construct a deeper understanding of physical concepts through step-by-step confrontation with their alternate conceptions.

6. We utilize techniques (such as the use of flash cards, show of hands, or electronic wireless transmitters) for acquiring immediate feedback from all the students in the class. (See Ref. 4.)

\section{Sample Interactive Demonstration}

In order to explore the physics of freely falling objects, a dime and a quarter are dropped simultaneously from the same height. The question to be answered is: "Which object would hit the floor first?" We then design an interactive demonstration sequence consisting of several conceptually linked mini-demonstrations to address important conceptual issues associated with free-fall. A set of multiple-choice questions for this demonstration sequence was developed for use with flash cards. Worksheets were designed on which students were required to write predictions and draw motion diagrams. Excerpts from the questions and worksheets are shown below.

\section{Initial Flash-Card Question}

A dime and a quarter are dropped simultaneously from the same height. Which one will hit the floor first?
A. The dime will hit the floor first.
$B$. The quarter will hit the floor first.
C. Both hit the floor at the same time.
D. I am not surel I don't know.

Students are always required to make predictions of the outcome of the demonstration by holding up flash cards, or using wireless electronic transmitters. They may "vote" before and/or after talking to their neighbors. At the appropriate times, the instructor will provide assistance. Once the first demonstration is complete and students have finished their discussions and worksheet activities, the process is continued by asking (one by one) seven other closely related questions.

\section{Follow-up Questions}

These questions all follow the model of the first one, but in each case different pairs of objects are compared. (Each question is accompanied by a separate worksheet for student responses). The questions ask students to compare the rates of fall of the following pairs of items: (1) dime and piece of paper; (2) dime and piece of crumpled paper; (3) coffee filter and loaded coffee filter; (4) book and piece of paper; (5) piece of paper resting on top of book; (6) dime and piece of paper inside an open chamber; (7) dime and piece of paper inside closed, evacuated chamber. 


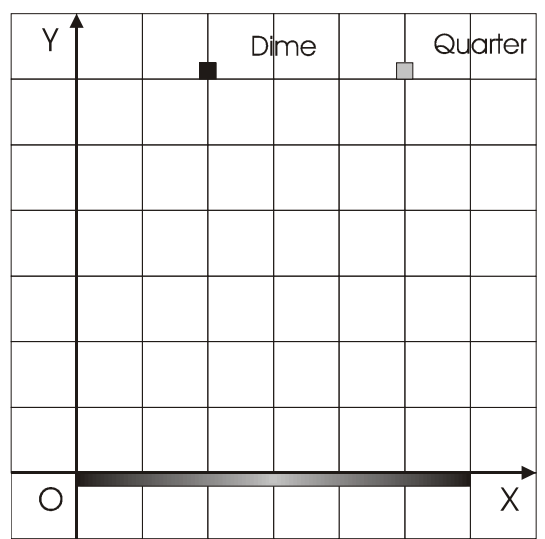

(1) A dime and a quarter are dropped simultaneously from the same height. Which one will hit the floor first?

A. The dime will hit the floor first.

B. The quarter will hit the floor first.

C. Both hit the floor at the same time.

D. I am not sure/I don't know.

[Most of the following instructions were given either verbally or written on the blackboard:]

Before the demonstration: In the space below, write down your prediction about the outcome of the demonstration. Write a few sentences to justify/explain your thinking.

After seeing the demonstration [sometimes, before seeing]: Draw motion diagrams to represent the motion of the dime and the quarter. Do this directly on the diagram above.

Fig. 1. Sample activity and worksheet excerpts.

\section{ANALYSIS OF CLASS-TEST DATA}

The method described here has been implemented twice in the context of an introductory algebra-based mechanics class. Although the available assessment data are limited and inconclusive, they do suggest the possibility that significant improvements might be ascribed to interactive demonstrations.

Here we focus specifically on an analysis of students' responses to Question \#1 on the Force Concept Inventory. This question targets precisely the concept that is the subject of the sample materials presented in this paper. For reference, we cite FCI \#1:

Two metal balls are the same size but one weighs twice as much as the other. The balls are dropped from the roof of a singlestory building at the same instant. The time it takes the balls to reach the ground below will be
A. about half as long for the heavier ball as for the lighter one.
B. about half as long for the lighter ball as for the heavier one.
C. about the same for both balls.
D. considerably less for the heavier ball, but not necessarily half as long.
E. considerably less for the lighter ball, but not necessarily half as long.

The first author implemented interactive demonstrations as described here for the first time during Fall 1998 at Southeastern Louisiana University (SLU). They were also used during Summer 2000 at Southwest Missouri State University (SMS). Previously, he had taught the same course (algebra-based mechanics) three times; twice at SLU and once at the University of Virginia (UVa). Table I presents data from all five of these classes.

Table I. Assessment data (mean pre- and posttest scores, and Hake normalized gains $<g>$ ) for algebra-based mechanics courses taught by first author. Courses in boldface (SLU 98 and SMS 00) used fully structured interactive demonstration (described in this paper) for free-falling objects. The other courses used more limited demonstrations involving only straightforward predictions.

\begin{tabular}{|c|c|c|c|c|c|}
\hline & $n$ & $\begin{array}{l}\text { FCI } \\
\# 1 \\
\text { pre }\end{array}$ & $\begin{array}{l}\text { FCI } \\
\# 1 \\
\text { post }\end{array}$ & $\begin{array}{l}\text { FCI } \\
\# 1 \\
<g>\end{array}$ & $\begin{array}{l}\text { Full } \\
\text { FCI } \\
<g>\end{array}$ \\
\hline UVa 95 & 55 & $71 \%$ & $82 \%$ & 0.38 & 0.26 \\
\hline SLU 96 & $75 *$ & $57 \%$ & $78 \%$ & 0.49 & 0.15 \\
\hline SLU 97 & 66 & $79 \%$ & $85 \%$ & 0.29 & 0.30 \\
\hline SLU 98 & 31 & $65 \%$ & $100 \%$ & 1.00 & 0.34 \\
\hline SMS 00 & 22 & $36 \%$ & $91 \%$ & 0.86 & $\mathbf{0 . 5 0}$ \\
\hline
\end{tabular}


All five of these classes were taught with interactive-engagement methods. In particular, they made heavy use of highly interactive "lectures" using the flash-card method. (These methods have been described in detail elsewhere; see Reference 4.) With specific regard to the concept of a freely falling object, there were significant differences in the instructional method used.

A full implementation of interactive demonstrations as described here, with a full sequence of conceptually linked questions, was used only for the SLU 98 and SMS 00 courses. The other courses used a format in which students' predictions were, indeed, solicited before the demonstration took place. However, in those cases there was little or no attempt to structure a series of tightly linked interactive demonstrations with a single theme as we have described here. (One of the consequences of using the full interactive method is that several additional minutes are required for the activity.)

This full implementation was only carried out for the concept of the free-falling object, and that is why data only for FCI \#1 are examined in this section. The most remarkable result is that every single one of the 31 students in SLU 98 got that question correct on the posttest, while only $65 \%$ had it correct on the pretest. This performance was far better than that of SLU 97 or SLU 96. The $100 \%$ posttest score is significantly better than the $85 \%(p=0.02)$ of SLU 97, the $78 \%(p=0.004)$ of SLU 96, and the $82 \%(p=0.01)$ of UVa, even though the pretest score for SLU 98 is relatively low. Although the overall FCI normalized gain of 0.34 for SLU 98 is nearly the same as that for SLU 97, its $<g>$ for FCI \#1 is far higher. This very high gain for FCI \#1 is nearly matched by the SMS 00 course, which also used the interactive demonstration method.

\section{SUMMARY}

We have described a method for implementing classroom demonstrations in a highly interactive fashion to promote active learning. The key aspects of this method are (1) create a carefully structured sequence of conceptually linked demonstrations, and (2) promote students' active engagement with the demonstrations by soliciting their input on multiple-choice questions using a classroom communication system, such as flash cards. Preliminary data from classroom testing are promising and suggest that this method may be able to produce significant learning gains.

We believe that the "highly interactive" format may increase the pedagogical effectiveness of the demonstrations, as is suggested by the data in Table I. Since the same instructor employing interactiveengagement methods taught all five courses, it is unlikely that "teaching to the test" produced this increase. In this regard, we intend to examine correlations among performances on FCI questions 1, 3 and 13 (all related to free-fall). Although we do not now have student achievement or attitude data to correlate with FCI data, in the future we plan to investigate these variables. We plan to develop and test additional interactive demonstrations on other topics to further explore the potential of this method.

\section{REFERENCES}

1. Pamela A. Kraus (1997). Promoting Active Learning in Lecture-Based Courses: Demonstrations, Tutorials, and Interactive Tutorial Lectures. Ph.D. dissertation, U. Washington, UMI \#9736313.

2. J. P. Callan and E. Mazur, "Classroom demonstrations: education or mere entertainment?" AAPT Announcer 29(4), 89 (1999).

3. D. R. Sokoloff and R. K. Thornton, "Using interactive lecture demonstrations to create an active learning environment," Phys. Teach. 35(10), 340-347 (1997).

4. D. E. Meltzer and K. Manivannan, "Promoting interactivity in physics lecture classes," Phys. Teach. 34(2), $72-76$ (1996).

K.M. acknowledges the support of a USDEPT3 Implementation grant P342A99041 and FFT grants. 